\title{
Experimental Study of Perfectly Patterned Silica-Titania Optical Waveguide
}

\author{
Rimlee DEB ROY ${ }^{1}$, Devika SIL ${ }^{2,3}$, Sunirmal JANA ${ }^{2}$, Prasanta Kumar BISWAS ${ }^{2}$, and \\ Shyamal Kumar BHADRA ${ }^{1}$
}

\author{
${ }^{1}$ Fiber Optics \& Photonics Division, Central Glass \& Ceramic Research Institute, 196 Raja S. C. Mullick Road, \\ Jadavpur, Kolkata-700 032, India \\ ${ }^{2}$ Sol-Gel Division, Central Glass \& Ceramic Research Institute, 196 Raja S. C. Mullick Road, Jadavpur, \\ Kolkata-700 032, India \\ ${ }^{3}$ Department of Chemistry, Temple University, Philadelphia, PA, 19122, USA \\ *Corresponding author: Shyamal Kumar BHADRA_E-mail: skbhadra@cgcri.res.in
}

\begin{abstract}
Inorganic silica-titania thin films with thicknesses $150 \mathrm{~nm}-200 \mathrm{~nm}$ are deposited on high purity and polished silicon wafer and silica glass substrates by sol-gel dipping process and are patterned by capillary force lithography technique. Subsequently grating structure is embossed in green stage. The patterned gel films are subjected to stepwise heat treatment to $500{ }^{\circ} \mathrm{C}$ and above in pure oxygen atmosphere in order to achieve major conversion of mixed-gel to oxide optical films which are characterized by Ellipsometry, Fourier transform infrared spectroscopy (FTIR) and atomic force microscopy (AFM) to optimize the fabrication parameters and to get perfectly matched film. Removal of organics and formation of perfectly inorganic silica-titania network at optimized heat treatment in controlled environment are ensured by FTIR spectral study. The difference in refractive indices between the substrate and coated film as calculated theoretically matches exactly with the developed waveguides for operating wavelength $(632.8 \mathrm{~nm})$ and the measured optical properties show the planar waveguide behavior of the films.
\end{abstract}

Keywords: Optical properties, sol-gel technique, thin films, oxides

\section{Introduction}

Increasing interest in photonics integrated circuits has stimulated studies on newer optical materials. Sol-gel derived materials such as $\mathrm{SiO}_{2}$ and $\mathrm{TiO}_{2}$ doped with metal nano-particles have been investigated for various optical devices [1]. Sol-gel processed organic-inorganic composite materials are capable of producing thick enough films by single coating without cracking. For example, Makoto Yoshida et al. [2] reported an optical propagation loss of $0.62 \mathrm{~dB} / \mathrm{cm}$ or lower at $633 \mathrm{~nm}$ based on
$\mathrm{SiO}_{2} / \mathrm{TiO}_{2} /$ poly(vinylpyrrolidone) composite materials derived by sol-gel technique. The most extensively used optical waveguide is the step index optical fiber, but the simplest optical waveguide is the planar waveguide structure which consists of a thin dielectric film sandwiched between materials of slightly lower refractive indices [3]. Light wave introduced into the high index film or rectangular core arrives at the boundary at an angle which is greater than the critical angle of total internal reflection [4]. Interestingly, with the development of polymer waveguide devices, gratings have gained

Received: 5 March 2011/ Revised version: 22 March 2011

(C) The Author(s) 2011.This article is published with open access at Springerlink.com 
considerable interest in efficient coupling of light of particular diffracted mode into these waveguides for use as precision sensing device [5]. The technique for developing such devices has also been applied to inorganic systems as well for better structural stability and high temperature application. The synthesis of transparent $\mathrm{SiO}_{2}$ based sol-gel coatings has been of great interest in recent years since this process of using metal alkoxide solutions can be applied to various substrates by spin or dip coating and enables good control of composition and optical properties [6, 7]. It is capable of coating large surfaces and does not require high processing temperatures. Lukowiak et al. [8] showed a silica-titania waveguide with a grating coupler, but they used a polymer photoresist on which the grating structure was inscripted by holographic exposure. This is a cumbersome affair whereas the soft lithographic technique is much easier to process. Amongst others the sol-gel method is used to obtain thin film planar waveguides with good optical and mechanical properties, where in the process of dip-coating the substrate is withdrawn from the sol at a constant rate. The entrained film becomes thin by means of evaporation of solvent and gravitational draining. Because the shape of the depositing film remains constant with respect to the reservoir surface, it is possible to use analytical methods such as ellipsometry and fluorescence spectroscopy to characterize the depositing film in situ. The microstructure and properties of the film depend on the size and structure of the inorganic sol species, the magnitude of the capillary pressure exerted during drying, and the relative rates of condensation and drying. By controlling these parameters, it is possible to reduce the nano-porosity of the transparent film considerably over a wide range [9].

Recently inscription of grating in bulk glassy waveguides by ultrafast lasers has created another possibility of intricate design and fabrication. This process enables patterning with nanometer resolution, which is of great importance for manufacturing advanced electronic, optical and mechanical devices [10, 11]. However, current research focuses on printing processes other than conventional photolithography to increase the range of patterning applications in order to get diffraction limited output [10]. A number of lithographic techniques have been developed among which soft lithography [12, 13], which relies on reusable stamps, generally fabricated of (by Xia et al.) poly-dimethyl siloxane (PDMS), is used to fabricate high quality patterned micro- and nano-structures directly onto gel-films. Along with soft patterning process, capillary force lithography (CFL) has proven to be fruitful [14-16]. It is a simple and effective tool for patterning, and it employs the concept of capillarity [17], where a polymer melt is molded by the use of an elastomeric stamp i.e. the PDMS stamp. We have used this soft lithographic technique to emboss grating structures on as-deposited silica-titania gel films of desired configurations for targeted operating wavelength of waveguide. It needs a state of certain visco-elastic stage of the gel-film suitable for embossing and structural stability. Subsequent heat treatment in controlled environment produces transparent oxide film with expected low optical loss.

Waveguides thus fabricated are subjected to different studies like the atomic force microscopy (AFM) scan, Ellipsometry, Fourier transform infrared spectroscopy (FTIR). Finally optical characteristics of the waveguide films are analyzed using polarized He-Ne laser. Grating couplers as sensor elements were for the first time introduced by a research team of Lukosz $[18,19]$. Later on this was also thoroughly investigated by Kuntz et al. [20, 21]. The high sensitivity can only be ensured by sensor structures produced with the application of homogeneous waveguides of high refractive indices. In this paper we study the physico-chemical processes and steps to develop highly transparent embossed grating waveguide coupler particularly aiming at the application in precision sensing. 


\section{Theory}

We have studied theoretically the design of silica-titania $\left(\mathrm{SiO}_{2}-\mathrm{TiO}_{2}\right)$ waveguides by taking real fabrication parameters into consideration. A simple planar waveguide consists of a film (as shown in Fig. 1) of refractive index $n_{\mathrm{f}}$ deposited on a substrate of refractive index $n_{\mathrm{s}}$ and a cover of refractive index $n_{\mathrm{c}}$. The confinement of light energy is along one transverse dimension and the light energy can diffract in the other transverse dimension.

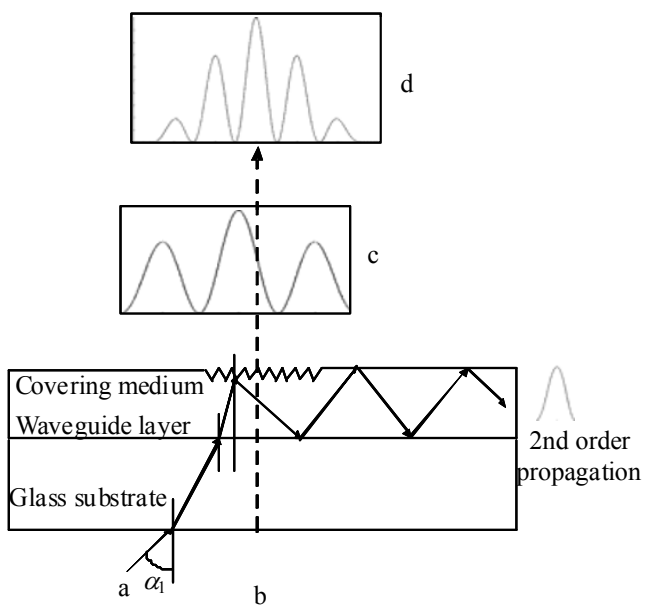

Fig. 1 Schematic representation of the grating coupler waveguide sensor.

The thin film waveguides are characterized by a refractive index variation of the form:

$$
n(x)=\left\{\begin{array}{l}
n_{\mathrm{c}}, \quad x>0, \quad \text { cover } \\
n_{\mathrm{f}},-d<x<0, \text { film } . \\
n_{\mathrm{s}}, \quad x<0, \text { substrate }
\end{array}\right.
$$

The above refractive index variation corresponds to a thin film of refractive index $n_{\mathrm{f}}$ and thickness $d$ deposited on a transparent substrate of refractive index $n_{\mathrm{s}}\left(n_{\mathrm{s}}<n_{\mathrm{f}}\right)$. The region above the film is referred to as the cover of refractive index $n_{\mathrm{c}}\left(n_{\mathrm{c}}<n_{\mathrm{f}}\right)$. In most cases, the cover is considered as air $\left(n_{\mathrm{c}}=1\right)$. Light guidance in such planar waveguide takes place on the basis of total internal reflection (TIR), hence we assume $n_{\mathrm{f}}>n_{\mathrm{s}} \geq n_{\mathrm{c}}$. For such one-dimensional waveguide, Maxwell's equations are reduced to two independent sets of equations. Both the transverse electric (TE) and transverse magnetic (TM) modes are equally probable to exist inside the planar waveguide. We know for guided modes the field should decay in the cover and substrate so that most of the energy associated with the mode lies inside the film. Hence for guided modes, we must have

$$
k_{0}^{2} n_{\mathrm{s}}^{2}<\beta^{2}<k_{0}^{2} n_{\mathrm{f}}^{2} \text {. }
$$

Using the boundary conditions and the continuity of $E_{y}$ and $d E_{y} / d x$ at $x=0$ and $x=-d$ we obtain the transcendental equation for the TE modes as [3]

$$
\tan \left[V(1-b)^{\frac{1}{2}}\right]=\frac{\left(\frac{b}{1-b}\right)^{\frac{1}{2}}+\left(\frac{b+a}{1-b}\right)^{\frac{1}{2}}}{1-\frac{[b(b+a)]^{\frac{1}{2}}}{(1-b)}}
$$

where

$$
\begin{gathered}
b=\frac{\beta^{2} / k_{0}^{2}-n_{\mathrm{s}}^{2}}{n_{\mathrm{f}}^{2}-n_{\mathrm{s}}^{2}}=\frac{n_{\mathrm{eff}}^{2}-n_{\mathrm{s}}^{2}}{n_{\mathrm{f}}^{2}-n_{\mathrm{s}}^{2}}, n_{\mathrm{eff}}=\frac{\beta}{k_{0}}, \\
V=k_{0} d\left(n_{\mathrm{f}}^{2}-n_{\mathrm{s}}^{2}\right)^{\frac{1}{2}}, \quad a=\frac{n_{\mathrm{s}}^{2}-n_{\mathrm{c}}^{2}}{n_{\mathrm{f}}^{2}-n_{\mathrm{s}}^{2}} .
\end{gathered}
$$

Similarly for TM modes we obtain a similar transcendental equation as

$$
\tan \left[V(1-b)^{\frac{1}{2}}\right]=\frac{\frac{1}{\gamma_{1}}\left(\frac{b}{1-b}\right)^{\frac{1}{2}}+\frac{1}{\gamma_{2}}\left(\frac{b+a}{1-b}\right)^{\frac{1}{2}}}{1-\frac{1}{\gamma_{1} \gamma_{2}} \frac{[b(b+a)]^{\frac{1}{2}}}{(1-b)}}
$$

where

$$
\gamma_{1}=\left(n_{\mathrm{s}} / n_{\mathrm{f}}\right)^{2}, \gamma_{2}=\left(n_{\mathrm{c}} / n_{\mathrm{f}}\right)^{2}=\gamma_{1}-a\left(1-\gamma_{1}\right) .
$$

By solving the two transcendental equations for TE and TM modes successively we are able to calculate a range of refractive indices and propagation constants of the guided modes and the corresponding film thicknesses. These are the main guidelines to be followed during the fabrication of the planar waveguide films in our experiment.

By assuming the values of $n_{\mathrm{c}}$ (air, 1.0), $n_{\mathrm{f}}(2.0)$ and $n_{\mathrm{s}}$ (1.46) we obtain a range of the film thicknesses where the waveguide would remain a single moded structure, i.e. propagation of the fundamental modes only. By varying the value of $d$ i.e. the film thicknesses (from $110 \mathrm{~nm}$ to $200 \mathrm{~nm}$ ) 
and using above (3), (4), and (5), a variation of the value of the refractive index of the guided mode can be obtained. A plot of the variation of the effective refractive index of the guided mode $\left(n_{\text {eff }}\right)$ and propagation constant $(\beta)$ with film thickness $(d)$ is shown in Fig. 2, which enables us to make a choice of the film thickness and the value of the film refractive index at a particular operating wavelength of light. From this graph we are able to select the refractive index and film thickness which we desire to obtain in our fabricated planar waveguides at $632.8 \mathrm{~nm}$ operating wavelength of light.

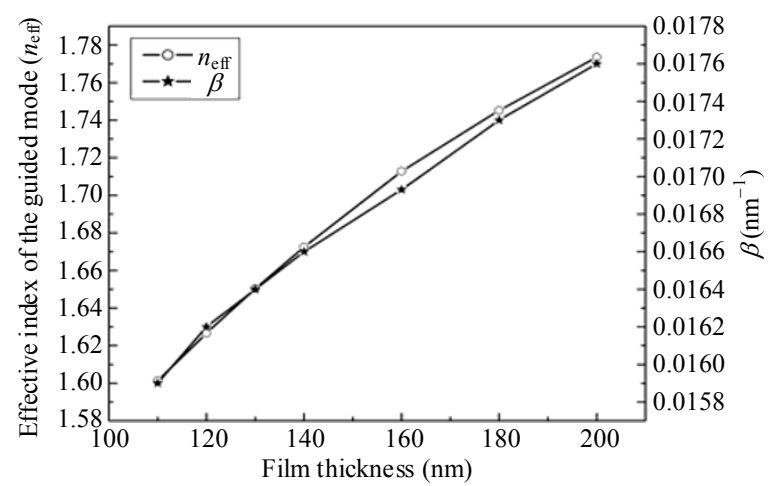

Fig. 2 Variation of the effective refractive index of the guided mode $\left(n_{\text {eff }}\right)$ and propagation constant $(\beta)$ with film thickness $(d)$.

The coupling of the light as shown in Fig. 1 into a waveguide by means of a grating coupler occurs at angles according to the following equation [14]:

$$
N=n \sin \left(\alpha_{l}\right)+l(\lambda / \Lambda)
$$

where $N=$ effective refractive index, $n=$ refractive index of air, $\alpha_{l}=$ coupling angle, $l=$ diffraction order, $\lambda=$ laser wavelength, $\Lambda=$ grating constant. For example, if the light of wavelength $632.8 \mathrm{~nm}$ is made incident on the waveguide at $60^{\circ}$ angle and then using the (7), the effective refractive index $(N)$ obtained is 1.7098 with second order diffraction $(l=2)$, which lies well within the range of $n_{\text {eff }}$ values as shown in Fig. 2. In Fig. 1, "d" shows the diffraction pattern as seen on a screen due to vertical incidence where the incident angle is $\alpha=0$ (demarcated by the "b" marked laser beam shown in Fig. 1). If the incident angle is changed to $\alpha=\alpha_{1}$ (indicated by the "a" marked laser beam shown in Fig. 1) the diffraction pattern is shown as "c" (in Fig. 1). The second order spot generated due to the diffraction is made to propagate through the waveguiding film by changing the incident angle to some value $\alpha_{1}$ which can be calculated from (7). We have been able to match this experimentally. Equation (7) makes the fact quite obvious that the accurate sensing requires a fine resolution of the incident angle which is easier if light source with small spectral line width is used.

\section{Fabrication and experimental details}

We have adopted sol-gel process in developing $\mathrm{SiO}_{2}-\mathrm{TiO}_{2}$ light-guiding layer. In order to emboss grating structure stamps of PDMS containing the negative replica of the patterns of a master grating have been used. For the preparation of the precursor of PDMS, weighted amounts of Sylgard Silicone Elastomer 184 and Sylgard 184 Curing Agent are thoroughly mixed and deaerated in a vacuum desiccator by exposing it to a low vacuum. After the solution becomes completely free of air bubbles it is poured onto the master grating mold and cured at $100{ }^{\circ} \mathrm{C}$ for 20 hours. Cross linking takes place by which the inner surface of the PDMS film develops the patterns as encrypted on the master mold. After the completion of curing of PDMS, small pieces are cut and used to emboss the desired patterns on the film surface. Each stamp has parallel stripes with periodicity of $1.5 \mu \mathrm{m}$ and stripe height of about $120 \mathrm{~nm}$. We have used the single step dip-coating process for the fabrication of the waveguide film on high purity and highly polished silica glass (Suprasil grade, Heraeus, Germany) and also on the polished surface of silicon wafers. The samples are then heated in step-wise manner at high temperatures following a heating schedule. Sol-gel process combines the advantage of thermal and optical power stability of inorganic materials for its easy formation from the inorganic-organic hybrid precursor. The mixed oxide films with refractive indices from 1.2 to 2 can be prepared at ease. The 
mere control of porosity of the matrix will lower the refractive index. As for example, this has been created in silica-titania thin films for its important applications as a component of anti-reflection coatings but more significantly as active and passive waveguides. A dispersion graph ( $b$ vs $V$ ) for one of the silica-titania waveguides fabricated by us is shown in Fig. 3, where $b$ is the normalized propagation constant and $V$ is the waveguide parameter described earlier. The curves for propagating TE and TM modes depicting single mode propagation are simulated using various parameters for the silica-titania sample fabricated by us. A three-layer (consisting of the cover, film and substrate) planar waveguide is considered. The cover layer is air of refractive index $\left(n_{\mathrm{c}}\right)$ 1.0, the waveguiding film layer is a silica-titania sol-gel film of refractive index $\left(n_{\mathrm{f}}\right) 1.7264$, and the substrate is a piece of silica glass with refractive index $\left(n_{\mathrm{s}}\right) 1.4573$ at $632.8 \mathrm{~nm}$. The film thickness can be fixed at any value between $93 \mathrm{~nm}$ and $434 \mathrm{~nm}$ in order to obtain single mode propagation. Figure 3 shows that below a certain value of $V$, called the cutoff frequency $\left(V_{c}\right)$, there are no modes in the system. For single mode propagation to take place the $V_{\mathrm{c}}$ values for TE and TM modes should be

$$
\begin{aligned}
& V_{\mathrm{c}}^{\mathrm{TE}}=\tan ^{-1} a^{\frac{1}{2}}+m \pi \quad \text { for TE modes } \\
& V_{\mathrm{c}}^{\mathrm{TM}}=\tan ^{-1}\left(a^{\frac{1}{2}} / \gamma_{2}\right)+m \pi \text { for TM modes }
\end{aligned}
$$

where $m=0,1,2, \ldots$ For the propagation of the fundamental mode $m=0$ the value of $V_{\mathrm{c}}^{\mathrm{TE}}$ and $V_{\mathrm{c}}^{\mathrm{TM}}$ can be calculated for a particular value of refractive index of the waveguiding film developed and for fixed value of refractive indices of the cover and the substrate. These are the most vital points to be taken care during the fabrication of the desired planar waveguide. In Fig. 3 we have shown the $b$ vs $V$ curve for both TE and TM modes in case of single mode propagation in the fabricated planar waveguide. Many such waveguides of identical design have been developed by us, out of which one of its kind is presented in Fig. 3.

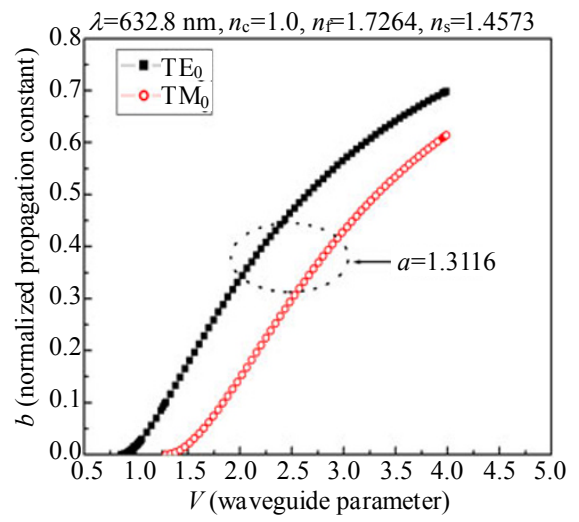

Fig. 3 Variation of the normalized propagation constant $b$ versus the waveguide parameter $V$ for TE and TM modes with asymmetry value $a=1.3116$.

The theoretical values thus have guided us to fabricate the desired planar waveguides. In Fig. 3, $a$ (calculated value of $a=1.3116$ ) is known as the asymmetry parameter and it may vary from zero to very large values for highly asymmetric waveguides.

Inorganic oxides based silica-titania planar waveguides can be produced with easy tunability of the refractive index and a loss lower than $1 \mathrm{~dB} / \mathrm{cm}$ [22]. We used Si (IV) and Ti (IV) alkoxides based composite sol layer on the substrate which turns to gel layer in due course of time. Tetra-isopropyl-orthotitanate (TIOT, purum grade, Fluka Chemika) and tetraethoxysilane (TEOS, $>98 \%$ purity, Fluka Chemika) were used as the starting precursor materials of titania and silica respectively during the preparation of the silica-titania sol. The detailed preparation can be found elsewhere [23]. The solvents used for the preparation of precursor sols were 2-butanol ( $\geq 99 \%$, E Merck India Ltd., for synthesis), 1-propanol $(\geq 99 \%$, E Merck India Ltd., for synthesis) and 2-propanol (E Merck India Ltd., for synthesis). Very dilute $\mathrm{HCl}$ (E Merck India Ltd., GR grade) was used as catalyst for the hydrolysis of TEOS, and acetylacetone $(\geq 98 \%$, E Merck India Ltd., for synthesis) was used as the complexing agent for controlling the fast hydrolysis rate of TIOT alkoxide. Double distilled water was used for the hydrolysis of TEOS. The substrates used for the dip coating were 
highly polished pure silica glass and silicon wafer. All the substrates were cleaned following the steps as reported elsewhere [24]. After 3 hours of preparation of the silica-titania sol, the substrates were coated by single step dip coating technique, with a lifting speed of $16 \mathrm{~cm} / \mathrm{min}$.

Patterning of the sol gel films is done by embossing small pieces of the elastomeric stamp of PDMS on the dip coated film to obtain required pattern on the film surface. We have adopted the process of solvent assisted capillary force lithography instead of direct CFL. Here the PDMS stamps have been presoaked in a diluted solution of the silica-titania sol for a minute prior to patterning. This subsequently leads to the retention of solvent in the PDMS surface. When the swollen PDMS comes into conformal contact with the dip coated thin film surface the solvent held up in the PDMS rises up along the vertical walls of the stamp due to capillary action and such stamps are then pressed against the gel film of silica-titania without allowing much time lapse between the coating process and the patterning process. The conformal contact between the PDMS stamp and the gel film is maintained for a couple of hours after which the stamp is peeled off. Once the PDMS stamp is removed the sample is shifted to a controlled furnace where it is heat-treated in pure oxygen atmosphere at temperatures ranging from $100{ }^{\circ} \mathrm{C}$ to $500{ }^{\circ} \mathrm{C}$ and in some cases above $500{ }^{\circ} \mathrm{C}$.

Reproducibility of making exact patterns onto the gel film by means of elastomeric stamps of PDMS having encrypted the desired grating structure at room temperature is ensured without application of external pressure. The critical aspect of the technique is to optimize the proper visco-elastic properties of the mixed sol where the capillary rise of the liquid sol under the confining stamp takes place. The films are kept in conformal contact with the stamp under certain conditions for a defined time period. We have noticed from the experiments performed so far that although the variation of the refractive index is nearly constant when the film is heated above $450{ }^{\circ} \mathrm{C}$ the film thickness continues to shrink. Experiments are carried out in order to study the extent of shrinkage of the waveguide film as a function of the increasing temperature. The process is repeated to get good quality films. In each step the oxide films are characterized by FTIR spectra, ellipsometry, AFM and later optical measurement ensures the desired performance.

The AFM is done using the AFM Multiview 3000, Nanonics Israel, AFM machine at different heating cycles, and the thickness and refractive index of the samples are measured using an Ellipsometer equipped with $\mathrm{He}-\mathrm{Ne}$ red laser of wavelength $632.8 \mathrm{~nm}$ (Gaertner Auto-Gain Ellipsometer, L-116 B). The FTIR spectra are recorded using a FTIR instrument (Nicloet 5700, USA) while the optical measurement is set up in our laboratory.

\section{Results and discussion}

The values of the thicknesses and refractive indices of some of the representative silica-titania planar waveguide samples are listed in Table 1. The high index contrast between the silica substrate and the film layer can be maintained by the use of the silica-titania mixed system. By varying the silica and titania alkoxide concentrations in the mixed sol greater tunability of index contrast can be obtained [8]. The thicknesses of the silica-titania films are measured precisely after subsequent heat treatment. As described a number of samples of silica-titania films are deposited on highly polished pure silica glass and silicon wafer substrates. At lower temperatures the films are porous in nature and therefore due to scattering we did not obtain consistent ellipsometry data. With progression of heating more compactness i.e., film shrinkage is observed and the variation of thickness is comparatively lessened. The shrinkage is about $33 \mathrm{~nm}$ by heat treatment in the temperature ranging from $150{ }^{\circ} \mathrm{C}$ to $450{ }^{\circ} \mathrm{C}$ as obtained from ellipsometric 
measurement which is in agreement with the results studied by AFM.

Table 1 Thicknesses and refractive indices of the samples coated with $10 \mathrm{wt} \%$ 50:50 silica-titania mixed sol at $16 \mathrm{~cm} / \mathrm{min}$ lifting speed.

\begin{tabular}{ccc}
\hline $\begin{array}{c}\text { Heating } \\
\text { temperature }\left({ }^{\circ} \mathrm{C}\right)\end{array}$ & $\begin{array}{c}\text { Physical thickness } \\
\text { range }( \pm 3)(\AA)\end{array}$ & $\begin{array}{c}\text { Refractive index range } \\
( \pm 0.003)(\text { R.I })\end{array}$ \\
\hline 150 & 1960 & 1.698 \\
250 & 1915 & 1.693 \\
350 & 1710 & 1.700 \\
450 & 1630 & 1.703 \\
\hline
\end{tabular}

Brusatin et al. [25] reported that silica-titania films prepared by single step sol-gel dip coating process were heat-treated at $500{ }^{\circ} \mathrm{C}$ and were found to be homogeneous with desired waveguide loss. On the other hand, spin-coated silica-titania films heat-treated in air at different temperatures between $300{ }^{\circ} \mathrm{C}$ and $900{ }^{\circ} \mathrm{C}$ for 60 minutes in each step were not sintered properly since micro-structural reconstruction depended on the heat time as reported by Martins et al. [26]. In our case the silica-titania films were heat-treated for maximum 12 hours in each temperature setting (in steps of 6 hours +6 hours) in oxygen atmosphere and the waveguide appeared to be sintered properly with the formation of proportionate oxides to get the desired refractive index value and retained the embossed grating structure. Szendro et al. [27] studied the visco-elastic properties of the films for their suitability for embossing grating structure. After preparation of the sol we gave certain gelation time to get appropriate condition for stamping and subsequently, as described the PDMS stamp [28] as shown in Fig. 4, exactly replicated the patterns of the master grating on to the gel film.

The AFM images of the patterned silica-titania films heat-treated at different temperatures are shown in Fig. 5.

The line scan images clearly indicate that the height of the grating structure in ambient condition, which is $60 \mathrm{~nm}$, shrinks when heat-treated at $100{ }^{\circ} \mathrm{C}$

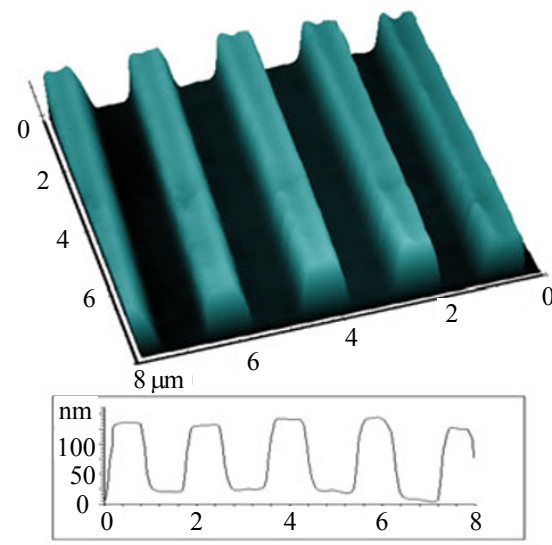

Fig. 4 AFM image of the PDMS stamp (Sylgard 184 stamp)(inset shows the cross sectional line profile).

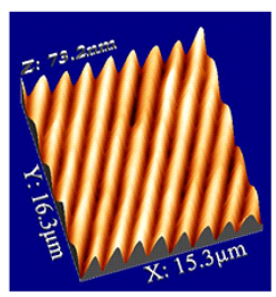

(a)

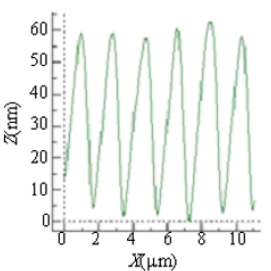

(d)

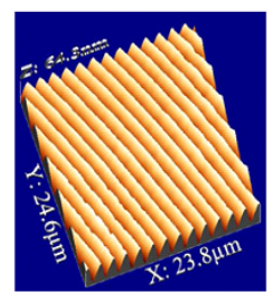

(b)

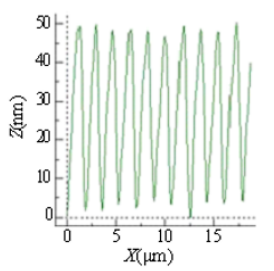

(e)

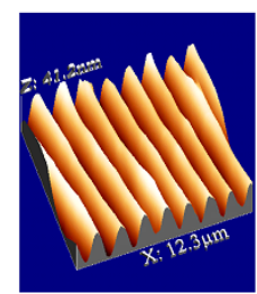

(c)

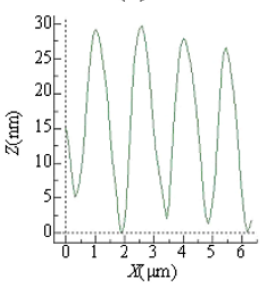

(f)
Fig. 5 (a) and (d) 3D AFM image of the grating in an as-coated (ambient) and patterned silica titania film and its corresponding line scan image, (b) and (e) AFM image of the grating in a patterned silica titania film and its corresponding line scan image cured at $100{ }^{\circ} \mathrm{C}$, (c) and (f) 3D AFM image of the grating in a patterned silica titania film cured at $450{ }^{\circ} \mathrm{C}$ and its corresponding line scan.

and shrinks further when heated subsequently at higher temperatures. The height of the embossed grating sample does not decrease considerably upon heating at $100{ }^{\circ} \mathrm{C}$, and the height obtained by AFM is about $50 \mathrm{~nm}$. The major shrinkage takes place when the film is heated to $450{ }^{\circ} \mathrm{C}$. We have heat-treated the samples at $500{ }^{\circ} \mathrm{C}$ and observed that the shrinkage is not significant and almost saturated when the samples are heated beyond $500{ }^{\circ} \mathrm{C}$. At 
$450{ }^{\circ} \mathrm{C}$ the height is about $27 \mathrm{~nm}$ which implies that shrinkage of about $55 \%$ has taken place. While there is shrinkage in the height of the grating patterns the peak to peak distance (pitch) remains almost unaltered with a separation of $1.5 \mu \mathrm{m}$. The film appears to be perfect for desired optical and waveguide properties.

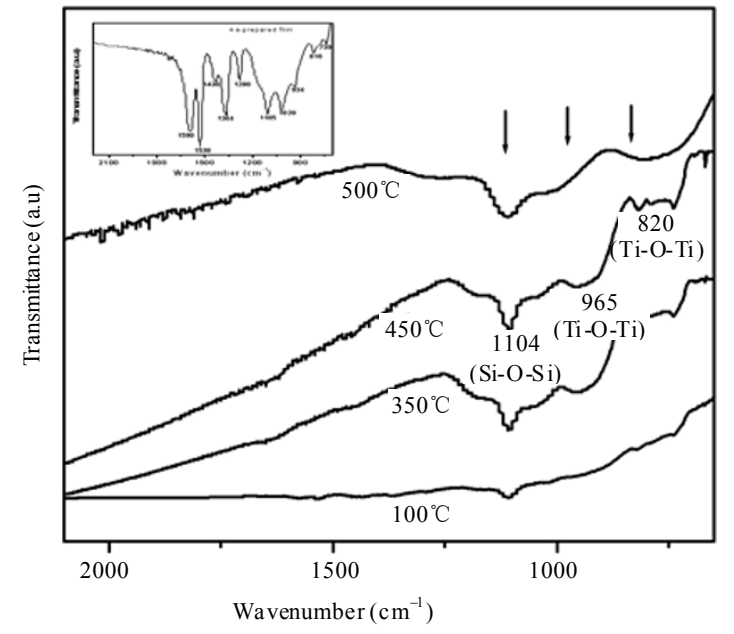

Fig. 6 FTIR spectra of the embossed silica titania films taken after curing at various temperatures (inset shows the FTIR spectra of the as-deposited (ambient film).

Figure 6 shows the substrate corrected FTIR spectra in the wavenumber region of $2000 \mathrm{~cm}^{-1}$ to $700 \mathrm{~cm}^{-1}$ of the silica-titania films on silicon wafer heat-treated at different temperatures in order to study the formation of inorganic oxide state. As-prepared (i.e. ambient) film shows prominent absorption peaks at $739 \mathrm{~cm}^{-1}, 818 \mathrm{~cm}^{-1}, 934 \mathrm{~cm}^{-1}$, $1020 \mathrm{~cm}^{-1}, 1105 \mathrm{~cm}^{-1}, 1260 \mathrm{~cm}^{-1}, 1364 \mathrm{~cm}^{-1}$, $1430 \mathrm{~cm}^{-1}, 1530 \mathrm{~cm}^{-1}$ and $1590 \mathrm{~cm}^{-1}$ (inset, Fig. 6). The spectra evolve due to the molecular vibrations in the region of $1600 \mathrm{~cm}^{-1}-1300 \mathrm{~cm}^{-1}$, which are the characteristic vibrations of actylacetonate complex of Ti(IV) [29]. The formation of this complex ensures the stability of Ti-alkoxide in the mixed sol system. It is known that asymmetric stretching vibrations of Si-O-Si bridging sequences appear in the range of $1300 \mathrm{~cm}^{-1}-1000 \mathrm{~cm}^{-1}$ [30], hence all the vibrations in this region are assigned to $\mathrm{Si}-\mathrm{O}-\mathrm{Si}$ asymmetric stretching vibrations [31]. It appears from the spectral curves that the short-wave shoulder around $1080 \mathrm{~cm}^{-1}$ becomes more pronounced with the increase of heating temperature. The absorption bands that appear at $818 \mathrm{~cm}^{-1}$ and $934 \mathrm{~cm}^{-1}$ are attributed to the presence of Ti-O-Ti and $[v(C-C)+v(C-O)]$ of Ti-actylacetonate complex and isolated silanol groups of hydrolyzed Si-alkoxide respectively [32]. With the increase of the baking temperature, intensity of the bands responsible for the Ti(IV)-actylacetonate complex becomes weak. When the baking temperature rises to $450{ }^{\circ} \mathrm{C}$, all the characteristic bands of Ti-actylacetonate complex disappear and a new band appears at $965 \mathrm{~cm}^{-1}$ due to the formation of Ti-O-Si [32] which is needed for homogeneous silica-titania oxide layer. On being heat-treated above $450{ }^{\circ} \mathrm{C}$, this band is much widened, indicating completion of oxide states of $\mathrm{SiO}_{2}$ and $\mathrm{TiO}_{2}$ significantly. Moreover, when the composite films are subjected to further annealing at higher temperatures it is found that the structure gets stabilized with little distortion in embossed structure which does not affect the optical properties. It is also evident from Fig. 6 that the wavenumber region of $1300 \mathrm{~cm}^{-1}-1000 \mathrm{~cm}^{-1}$ is broadened, indicating the Si-O-Si network enhancement in the film. Thus, increasing the temperature leads to the disappearance of the $\mathrm{CH}$ bands in the FTIR spectra and thus the removal of the $\mathrm{Si}-\mathrm{CH}_{3}$ bonds, as well as the reduction in the porosity of the film. This result confirms the densification of the film [6]. It is also observed that the films with embossed gratings which are heat-treated for a long period at $450{ }^{\circ} \mathrm{C}$ in a controlled environment have retained the desired structures required for optical properties. It is therefore inferred from the experimental study that controlled heating over a long time yield good structural stability and less porosity since less scattering is observed in ellipsometry study.

An optical setup with polarized $\mathrm{He}-\mathrm{Ne}$ laser source has been used to view the diffraction patterns produced by the silica-titania embossed grating planar waveguides as shown in Fig. 7. We are in a 
state to make reproducible waveguides of desired configurations. With the help of a helium-neon laser of $632.8 \mathrm{~nm}$ wavelength at normal incidence, we obtain the diffraction pattern produced due to the gratings of one of the representative samples as shown in Fig. 7.

Figure 7 shows the experimental setup along

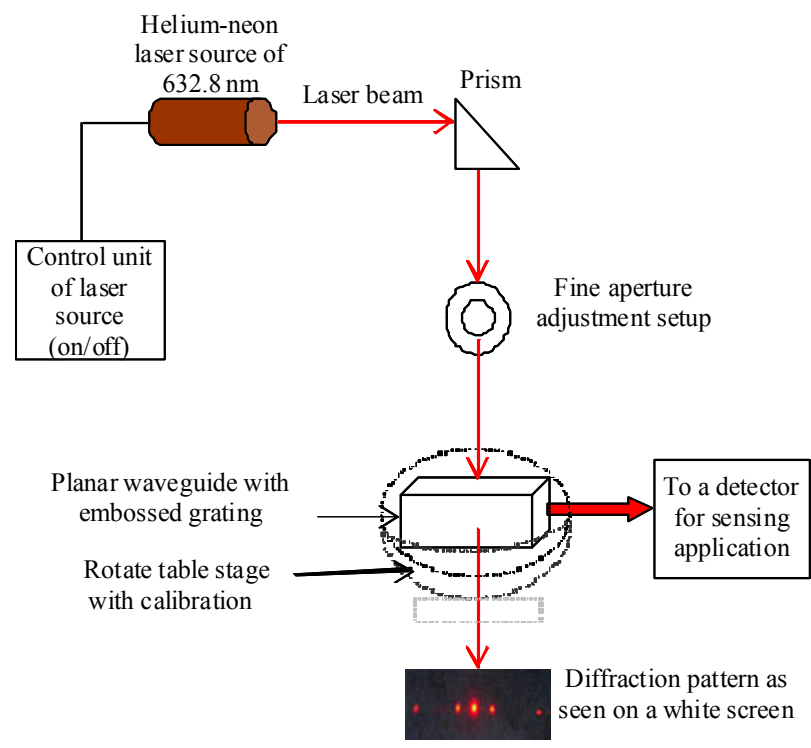

Fig. 7 Schematic of the experimental setup for optical characterization showing the diffraction pattern obtained from a silica-titania planar waveguide film at normal incidence.

with the second order diffraction pattern at normal incidence as seen on a screen from the waveguide sample. We have shown the coupling of the second order into the waveguide which propagates through the guiding film. Coupling of different diffraction orders into the waveguide can be studied by (7). We have calculated theoretically the incident angle by using (7) at which the second order propagates through the guiding film (as shown in Fig. 1). This result has been verified experimentally by placing the fabricated planar waveguide in the experimental setup. Figure 7 not only assesses the optical quality of the sol-gel embossed film [21], it also confirms that the fundamental spatial frequency of $666.67 \mathrm{~mm}^{-1}$ of the embossed grating is preserved during the drying and heat treatment of the sol-gel film derived from inorganic-organic hybrid precursor. The planar optical waveguide thus fabricated can be used suitably in sensing device.

\section{Conclusions}

Grating embossed silica-titania planar waveguide has been developed with good optical transparency and mechanical properties. The process of embossing of grating on the silica-titania sol-gel films in a controlled oxygen atmosphere and their subsequent heating to obtain perfect oxide sample, expands the field of application in optoelectronics and bio-sensing. The master grating used is low cost and easily available. The combination of fabricated PDMS and soft lithographic technique used has proved to be a successful process in such fabrications. But this is extremely dependent on visco-elastic property of the composite sol before stamping in order to maintain the grating structure after subsequent heat treatment for a long time and perhaps reported for the first time. The results obtained so far are promising and measures are being taken to obtain perfect waveguides so that they can be used as optical sensors.

\section{Acknowledgement}

The authors are grateful to Department of Science \& Technology (DST), Govt. of India, for financial grant and Director, Central Glass \& Ceramic Research Institute (CGCRI) for support and encouragement. The authors are also thankful to Dr. R. Mukherjee, presently in Indian Institute of Technology, Kharagpur (IIT-Kgp), India for his involvement at the early stage of the work and Dr. S. Bysakh for AFM studies.

Open Access This article is distributed under the terms of the Creative Commons Attribution License which permits any use, distribution, and reproduction in any medium, provided the original author(s) and source are credited. 


\section{References}

[1] W. Que, Y. Zhou, Y. L. Lam, Y. C. Chan, H. T. Tan, T. H. Tan, and C. H. Kam, "Sol-gel processed silica/titania/ÿ - glycidoxypropyltrimethoxysilane composite materials for photonics applications," J. Electron. Mater., vol. 29, no. 8, pp. 1052-1058, 2000.

[2] M. Yoshida and P. N. Prasad, "Sol-gel-processed $\mathrm{SiO}_{2} / \mathrm{TiO}_{2} /$ poly(vinylpyrrolidone) composite materials for optical waveguides," Chem. Mater., vol. 8, no. 1, pp. 235-41, 1996.

[3] A. Ghatak and K. Thyagarajan, Optical Electronics. UK: Cambridge University Press (reprinted in 2003), 1989, Chapters 11 and 14.

[4] C. J. Brinker and A. J. Hurd, "Fundamentals of sol-gel dip-coating," Journal de Physique III, vol. 4, no. 7, pp. 1231-1242, 1994.

[5] R. Waldhausl, B. Schnabe, P. Dannberg, E. B. Kley, A. Brauer, and W. Karthe, "Efficient coupling into polymer waveguides by gratings," Appl. Opt., vol. 36, no. 36, pp. 9383-9390, 1997.

[6] W. Que and X. Hu, "Optical and mechanical properties of sol-gel silica-titania hard optical coatings derived from methyltrimethoxysilane and tetrapropylorthotitanate as precursors," Opt. Mater., vol. 22, no. 1, pp. 31-37, 2003.

[7] R. Mizutani, Y. Oono, J. Matsuoka, H. Nasu, and K. Kamiya, "Coating of polymethylmethacrylate with transparent $\mathrm{SiO}_{2}$ thin films by a sol-gel method," J. Mater. Sci., vol. 29, no. 21, pp. 5773-5778, 1994.

[8] A. Lukowiak, R. Dylewicz, S. Patela, W. Strek, and K. Maruszewski, "Optical properties of $\mathrm{SiO}_{2}-\mathrm{TiO}_{2}$ thin film waveguides obtained by the sol-gel method and their applications for sensing purposes," Opt. Mat., vol. 27, no. 9, pp. 1501-1505, 2005.

[9] E. Hild, "Planar wave guides as chemical and biological sensors," accessed September 2009: http://www.microvacuum.com/pdf/products/biosensor /theory.pdf.

[10] X. Yu, Z. Wang, R. Xing, S. Luan, and Y. Han, "Solvent assisted capillary force lithography," Polymer, vol. 46, no. 24, pp. 11099-11103, 2005.

[11] H. Sirringhaus, N. Tessler, and R. H. Friend, "Integrated optoelectronic devices based on conjugated polymers," Science, vol. 280, no. 5370, pp. 1741-1744, 1998.

[12] N. Tessler, N. T. Harrison, and R. H. Friend, "High peak brightness polymer light-emitting diodes," $A d v$. Mater., vol. 10, no. 1, pp. 64-68, 1998.

[13] Y. Xia and G. M. Whitesides, "Soft lithography," Annu. Rev. Mater. Sci., vol. 28, no. 1, pp. 153-184, 1998.

[14] K. Tiefenthaler and W. Lukosz, "Sensitivity of grating couplers as integrated-optical chemical sensors," J. Opt. Soc. Am. B, vol. 6, no. 2, pp. 209-220, 1989.

[15] K. Y. Suh and H. H. Lee, "Capillary force lithography: large-area patterning, self-organization and anisotropic dewetting," Adv. Funct. Mater., vol. 12, no. 6+7, pp. 405-413, 2002.

[16] D. Y. Khang and H. H. Lee, "Pressure-assisted capillary force lithography," Adv. Mater., vol. 16, no. 2, pp. 176-179, 2004.

[17] W. Lukosz and K. Tiefenthaler, "Embossing technique for fabricating integrated optical components in hard inorganic waveguiding materials," Optics Lett., vol. 8, no. 10, pp. 537-539, 1983.

[18] K. Heuberger and W. Lukosz, "Embossing technique for fabricating surface relief gratings on hard waveguides," Appl. Opt., vol. 25, no. 9, pp. 1499-1504, 1986.

[19] K. Tiefenthaler and W. Lukosz, "Integrated optical switches and gas sensors," Optics Lett., vol. 10, no. 4, pp. 137-139, 1984.

[20] R. E. Kunz, J. Dubendorfer, and R. H. Morf, "Finite grating depth effects for integrated optical sensors with high sensitivity," Bios. Bioelectron., vol. 11, no. 6/7, pp. 653-667, 1996.

[21] R. E. Kunz, "Gradient effective index waveguide sensors," Sensors. Actuators B, vol. 11, no. 1-3, pp. 167-176, 1993.

[22] M. Montagna, E. Moser, F. Visintainer, M. Ferrari, L. Zampedri, A. Martucci, M. Guglielmi, and M. Ivanda, "Nucleation of titania nanocrystals in silica titania waveguides," J. Sol. Gel. Sci. Tech., vol. 26, no. 1-3, pp. 241-244, 2003.

[23] P. K. Biswas, D. Kundu, and D. Ganguli, “A sol-gel derived antireflective coating on optical glass for near-infrared applications," J. Mat. Sci. Lett., vol. 8, pp. 1436-1437, 1989.

[24] A. K. Atta, P. K. Biswas, and D. Ganguli, "A sol-gel derived yellow-transmitting coating on glass," $J$. Non-Cryst. Solids, vol. 125, no. 3, pp. 202-207, 1990. 
[25] G. Brusatin, M. Guglielmi, P. Innocenzi, A. Martucci, G. Battaglin, S. Pelli, and G. Righini, "Microstructural and optical properties of sol-gel silica-titania waveguides," J. Non-Cryst. Solids, vol. 220, no. 2-3, pp. 202-209, 1997.

[26] O. Martins and R. M. Almeida, "Sintering anomaly in silica-titania sol-gel films," J. Sol. Gel. Sci. Tech., vol. 19, no. 1-3, pp. 651-655, 2000.

[27] I. Szendro, "Art and practice to emboss gratings into sol-gel waveguides," Proc. SPIE, vol. 4284, pp. 80-87, 2001.

[28] M. Gonuguntla, A. Sharma, R. Mukherjee, and S. A. Subramaniam, "Control of self-organized contact instability and patterning in soft elastic films," Langmuir, vol. 22, no. 16, pp. 7066-7071, 2006.

[29] K. Schrijnemakers and E. F. Vansant, "Preparation of titanium oxide supported MCM-48 by the designed dispersion of titanylacetylacetone," J. Porous Mater., vol. 8, no. 2, pp. 83-90, 2001.

[30] S. Jana, M. A. Lim, I. C. Baek, C. H. Kim, and S. II Seok, "Non-hydrolytic sol-gel synthesis of epoxysilane-based inorganic hybrid," Mat Chem Phys., vol. 112, no. 3, pp. 1008-1014, 2008.

[31] D. Akram, S. Ahmad, E. Sharmin, and S. Ahmad, "Silica reinforced organic-inorganic hybrid polyurethane nanocomposites from sustainable resource," Macromol. Chem. Phys., vol. 211, no. 4, pp. 412-419, 2010.

[32] P. Cheng, M. P. Zheng, Y. P. Jin, Q. Huang, and M. Y. Gu, "Preparation and characterization of silica-doped titania photocatalyst through sol-gel method," Mater. Lett., vol. 57, no. 20, pp. 2989-2994, 2003. 